\title{
Development of Rheumatoid Arthritis During Anti-Interleukin-5 Therapy in a Patient with Refractory Chronic Eosinophilic Pneumonia
}

\author{
Hiroki Kawabata' \\ Minoru Satoh ${ }^{2}$ \\ Kazuhiro Yatera' \\ 'Department of Respiratory Medicine, \\ University of Occupational and \\ Environmental Health, Kitakyushu, \\ Fukuoka, 807-8555, Japan; ${ }^{2}$ Department \\ of Clinical Nursing, University of \\ Occupational and Environmental Health, \\ Kitakyushu, Fukuoka, 807-8555, Japan
}

\begin{abstract}
Purpose: To report the case of a patient with refractory chronic eosinophilic pneumonia who developed rheumatoid arthritis during anti-interleukin (IL)-5 therapy.

Case Report: The case of a 66-year-old male ex-smoker with allergic rhinitis who had dyspnea and chronic cough for 6 months and who was ultimately diagnosed with chronic eosinophilic pneumonia is reported. Long-term corticosteroid therapy was necessary due to recurrence of the chronic eosinophilic pneumonia during tapering of the corticosteroid. As a steroid sparing strategy, mepolizumab was initiated, and the steroid was tapered gradually. When the dose of prednisolone was $2 \mathrm{mg} /$ day, he developed polyarthralgia. Mepolizumab was changed to benralizumab considering the possibility that arthralgia was a side effect of mepolizumab; however, the arthralgia continued and he was ultimately diagnosed with rheumatoid arthritis. Methotrexate was initiated and his arthritis improved. Thereafter, benralizumab was discontinued after 5 injections, and he subsequently required neither systemic corticosteroids nor biologics.

Conclusion: The present case may suggest that suppression of IL-5 induces rheumatoid arthritis in certain patients; however, it is also possible that initial steroid therapy improved subclinical RA and made it remain undiagnosed, and the parallel OCS tapering during IL-5 therapy could have contributed to unveil the underlying RA. Further studies are required to establish guidelines on the optimum use of anti-IL-5 therapy and to understand the interactions between chronic eosinophilic pneumonia, anti-IL-5 therapy, tapering of corticosteroid and development of rheumatoid arthritis.
\end{abstract}

Keywords: asthma, chronic eosinophilic pneumonia, rheumatoid arthritis, mepolizumab, benralizumab

\section{Introduction}

Humanized monoclonal antibody to interleukin-5 (IL-5; mepolizumab) or the $\alpha$-chain of the IL-5 receptor (benralizumab) is effective treatment for eosinophilic asthma, chronic eosinophilic pneumonia (CEP), and other eosinophilic diseases. ${ }^{1}$ However, development of arthritis is a potential concern given that the consequent suppression of allergic Th2 cytokines would shift the cytokine balance toward Th1- and Th17-associated responses, which can lead to various inflammatory diseases, including rheumatoid arthritis (RA). ${ }^{2}$ Here, we describe the case of a patient with CEP who was treated with long-term oral glucocorticoid and developed RA during anti-IL-5 therapy and tapering of the glucocorticoid. Initial corticosteroid treatment might have suppressed RA symptom to make it remained undiagnosed and tapering of corticosteroid might help unmasking RA. Further
Correspondence: Hiroki Kawabata Department of Respiratory Medicine, University of Occupational and Environmental Health, I-I Iseigaoka, Yahata-nishiku, Kitakyushu, Fukuoka, 807-8555, Japan

Tel +8I-93-69|-7453

Fax +8I-93-602-9373

Email hirokik@med.uoeh-u.ac.jp 
investigation is needed to understand the role of anti-IL-5 therapy in the development of RA.

\section{Case Presentation}

A 66-year-old man with a history of smoking and allergic rhinitis for the last 14 years presented at our clinic in May 2012 due to dyspnea and productive cough lasted for 6 months in spite of treatment with inhaled fluticasone $(1000 \mu \mathrm{g} /$ day $) /$ salmeterol $(50 \mu \mathrm{g} /$ day $)$. His chest X-ray in October 2012 showed multiple infiltrative shadows (Figure 1A). A chest computed tomography revealed patchy infiltrations with ground glass attenuations predominantly around the pleura with air bronchograms inside in the left upper lobe (Figure 1B). A physical exam revealed
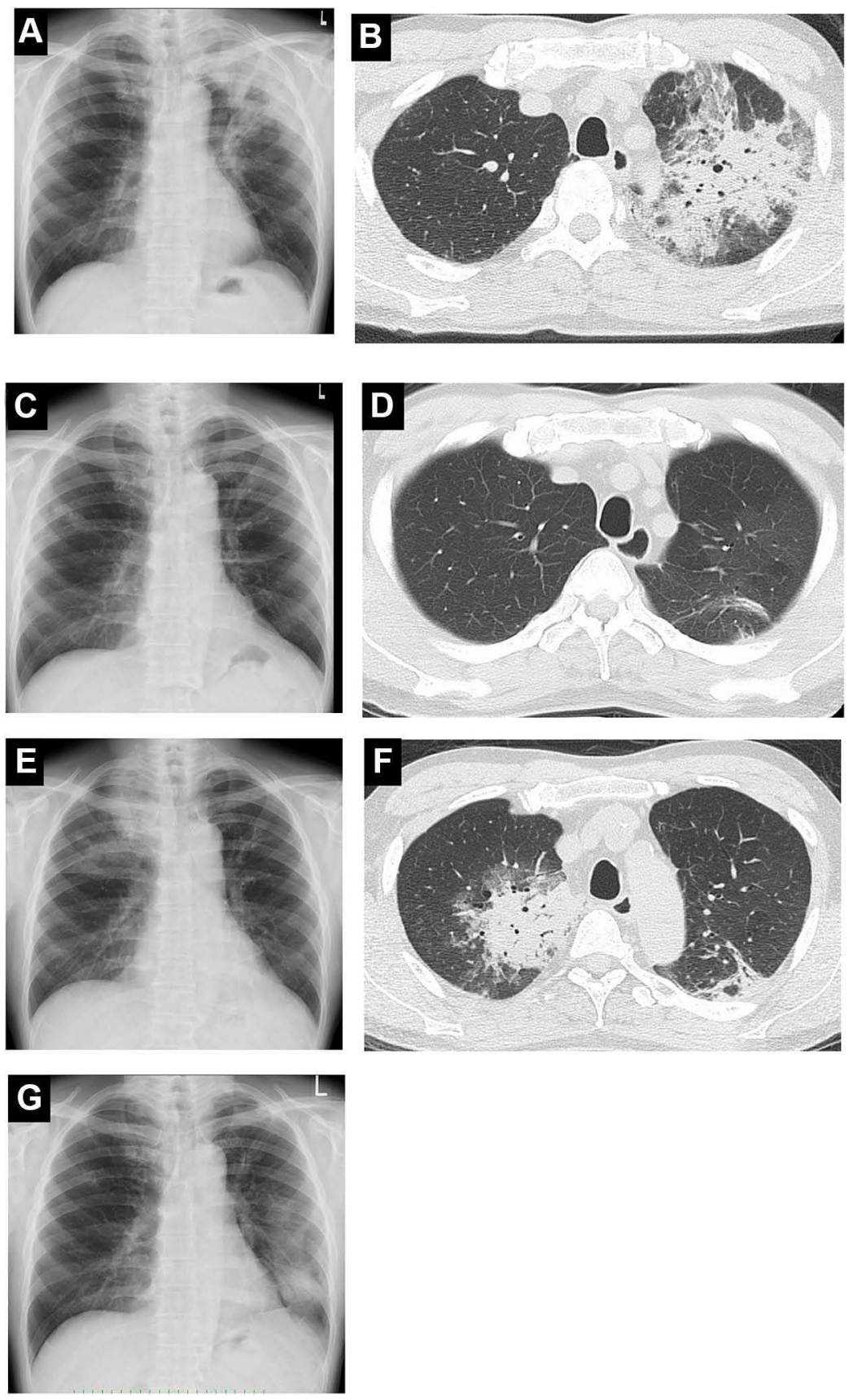

Figure I (A, B) Chest X-ray and chest CT scan in October 2012. Patchy infiltrations predominantly around the pleura with air bronchograms in the left upper lobe are seen. (C, D) Chest X-ray and chest CT scan in February 2013. Dramatic improvement is seen. (E, F) Chest X-ray and chest CT scan in April 20I3. Relapse of CEP showing infiltration in right upper lobe is shown. (G) Chest X-ray in August 2015. Infiltrative shadow in left lower lung field is seen.

Abbreviation: CT, computed tomography. 
wheezing in all lung fields. He had neither nasal polyposis nor signs for atopic dermatitis or peripheral neuropathy. His white blood cell count was $14,600 / \mu \mathrm{L}$ with eosinophil count of $5,563 / \mu \mathrm{L}$ (38.1\%). Serum immunoglobulin E (IgE) was 2,356 IU/mL (normal value $<175$ ) and Candida albicans specific IgE was positive (1.06 UA/ $\mathrm{mL}$, normal value $<0.34$ ) but all other specific IgE antibodies were negative. His serum showed no evidence of parasitic infection, and was negative for antinuclear antibodies, myeloperoxidase-specific antineutrophil cytoplasmic antibodies, and anti-cyclic citrullinated peptide antibodies $(<0.5 \mathrm{U} / \mathrm{m}$, normal value $<5)$, while positive for rheumatoid factor (RF, $26.2 \mathrm{IU} / \mathrm{mL}$, normal value $<15$ ). Bronchoalveolar lavage fluid (BALF) from the left upper lobe was predominantly eosinophilic (69.3\%) without significant pathogens. A transbronchial lung biopsy from the left upper lobe revealed eosinophilic inflammation without evidence of vasculitis or granuloma. Bone marrow aspiration was performed and was negative for malignancy and the FIP1L1-PDGFR fusion gene.

After ruling out other known causes of eosinophilic pneumonias, a diagnosis of CEP was made based on the duration of clinical symptoms ( $>2$ weeks), abnormal chest radiographic findings, eosinophilia, and evident eosinophilic infiltration in the lung. ${ }^{3}$ His respiratory symptoms quickly resolved with prednisolone (PSL) therapy but relapsed in April 2013. BALF from the right upper lobe was eosinophilic $(16.9 \%)$ despite oral prednisolone therapy. Continuous prednisolone therapy was necessary because of 2 flares including abovementioned flare that occurred during the tapering (Figure 2). In September 2017, subcutaneous injection of mepolizumab (100 mg every 4 weeks) was initiated to control CEP and to reduce the corticosteroid dose; PSL was tapered by 1 to $2 \mathrm{mg}$ per 4 weeks without worsening of respiratory symptom; however, in January 2018, after 5 injections with

inhalation therapy

Fluticasone $1000 \mu \mathrm{g} /$ Salmeterol $50 \mu \mathrm{g}$

biologic preparation

Mepolizumab Benralizumabb
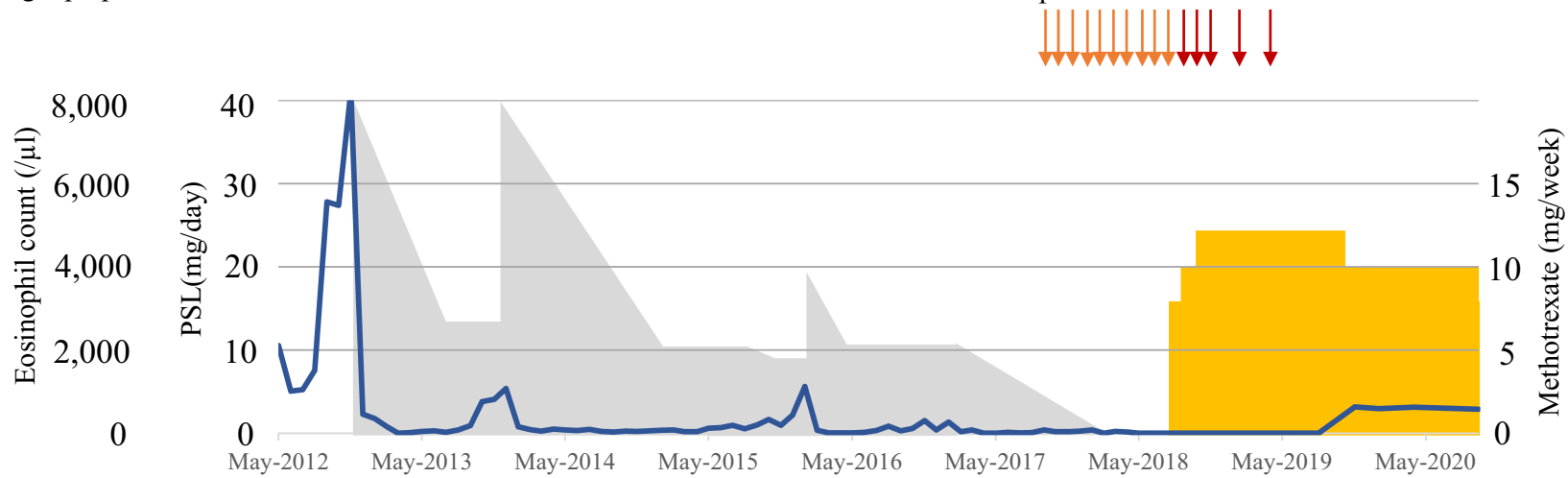

— Eosinophil counts $(/ \mu \mathrm{L})$

PSL (mg/day)

Methotrexate (mg/week)

\begin{tabular}{|c|c|c|c|c|c|c|c|c|c|c|c|c|c|}
\hline \multicolumn{14}{|c|}{ Respiratory symptom* } \\
\hline \multicolumn{14}{|l|}{ Arthritis** } \\
\hline $\mathrm{CRP}(\mathrm{mg} / \mathrm{dl})$ & 0.05 & 14.13 & 9.13 & 0.08 & 0.02 & 0.7 & 0.06 & 0.04 & 0.11 & 0.8 & 0.2 & 0.1 & 0.03 \\
\hline $\operatorname{ESR}(\mathrm{mm} / \mathrm{h})$ & 10 & 69 & 71 & 10 & & & 8 & 10 & 16 & 40 & 17 & 14 & 10 \\
\hline \multicolumn{14}{|c|}{ Pulmonary function } \\
\hline FEV1, 1 & 1.36 & 2.35 & & 19 & & & & & & 3.38 & & & \\
\hline FEV1/FVC, \% & 51 & 62 & & 80 & & & & & & 84 & & & \\
\hline
\end{tabular}

Figure 2 Clinical course of the patient. Oral prednisolone to control CEP was tapered off during mepolizumab therapy. Methotrexate was initiated for rheumatoid arthritis and mepolizumab was replaced by benralizumab. No exacerbations were noted after the discontinuation of benralizumab. The degree of respiratory symptom $(*$ green) and arthritis (**blue) are indicated.

Abbreviations: CEP, chronic eosinophilic pneumonia; CRP, C-reactive protein; ESR, erythrocyte sedimentation rate; FVC, forced vital capacity. 
mepolizumab and during PSL tapering at the dose of $2 \mathrm{mg} /$ day, he developed polyarthralgia and joint stiffness. In May 2018, PSL was tapered off without worsening of respiratory symptom. His arthralgia in bilateral proximal interphalangeal joints continued but he was not treated as RA because signs of active synovitis were absent and his hand X-ray did not show bone erosion. However, his arthritis gradually exacerbated and the number of affected joints increased. Finally in July 2018, a diagnosis of RA was made based on the criteria of American College of Rheumatology/European League Against Rheumatism; namely, arthritis in 8 small joints, positive rheumatoid factor $(22.6 \mathrm{IU} / \mathrm{mL})$, and articular symptoms lasting longer than 6 weeks. Methotrexate was started at $8 \mathrm{mg} /$ week and increased to $12 \mathrm{mg} /$ week. In August 2018, his treatment was switched from mepolizumab (total of 10 injections) to benralizumab (initial 2 doses, $30 \mathrm{mg}$ every 4 weeks; subsequently, $30 \mathrm{mg}$ every 8 weeks) because it was difficult to completely rule out the possibility that his arthritis was an adverse reaction related to mepolizumab. Meanwhile, his arthritis gradually resolved with methotrexate. In May 2019, benralizumab was discontinued after 5 injections because no respiratory symptoms were noted during benralizumab therapy. Although his eosinophil count increased from $0 \%$ to $\sim 10 \%$ at 3 months after the last benralizumab injection, no respiratory symptoms appeared for more than a year.

\section{Discussion}

The clinical course of this patient suggests a potential interaction between CEP, anti-IL-5 therapy, tapering of corticosteroids and RA because the development of arthritis and a diagnosis of RA occurred during anti-IL- 5 therapy and tapering of corticosteroid therapy. IL-5 is a classic Th2 cytokine associated with various allergic diseases and eosinophilic inflammation. It also is responsible for the maturation and release of eosinophils from the bone marrow, their activation, and elongation of their life span. Thus, anti-IL-5 therapy not only shifts the cytokine balance from allergic Th2 responses to inflammatory Th1 and Th17 responses, but it also significantly reduces eosinophils. All of these are in favor of development of RA. Additionally, emerging evidence indicated that eosinophils actively participate in the resolution of inflammation seen in RA. ${ }^{2}$ Data from animal models demonstrated the antiinflammatory effects of eosinophils in alleviating arthritis. $^{4,5}$ Thus, suppression of IL-5 or eosinophils by mepolizumab or benralizumab therapy can lead to arthritis or arthralgia. A few small-scale retrospective studies on mepolizumab indeed reported arthralgia as one of the most common adverse events along with headache (20\%-30\%). However, double-blinded clinical trials showed a low incidence of arthralgia, with no differences between the treatment and placebo groups. ${ }^{6}$ A meta-analysis found no association between benralizumab and an increased prevalence of arthralgia. ${ }^{7}$ Nevertheless, development of RA with mepolizumab therapy was reported in a patient with hypereosinophilic syndrome. ${ }^{8}$ Benralizumab therapy exacerbated arthritis in another patient with severe eosinophilic asthma complicated by refractory RA. ${ }^{9}$ Although the asthma was well controlled in the latter patient, asthma and RA could be successfully managed by a combination of benralizumab and golimumab. ${ }^{9}$ Furthermore, one study that evaluated the impact of mepolizumab in patients with concomitant eosinophilic asthma and RA who had low disease activity for at least 6 months reported that 6 out of 8 patients with RA developed flares of arthritis after mepolizumab therapy. ${ }^{5}$ In the present case, the patient developed RA during mepolizumab therapy. The half-life of mepolizumab is approximately 20-36 days. ${ }^{10}$ Mepolizumab was injected 5 times every 4 weeks before the development of arthralgia, therefore, mepolizumab concentration probably reached the steady state and might cause side effects via cytokine imbalance in the present case. Another factor that might affect the development of arthritis is administration and tapering of oral prednisolone. When he was first diagnosed with CEP, he was positive for RF. Although RF is positive in $\sim 5 \%$ of healthy individual and only a fraction of RF positive individual develops RA, RF is known to precede the development of arthritis and RA. ${ }^{11}$ Thus, he might be predisposed to development of RA prior to treatment for CEP and the initiation of corticosteroid treatment might suppress the development of RA for a while, then anti-IL -5 therapy and the tapering of glucocorticoid might help uncover the natural course of development of arthritis.

Discontinuing biologics in asthma is usually challenging due to persistent eosinophilic inflammation; however, the interaction between anti-IL-5 therapy and RA in our patient could have resolved the eosinophilic inflammation, which then permitted discontinuation of both biologics and steroids. Nevertheless, anti-IL-5 therapy could have promoted the development of RA while suppressing eosinophilic inflammation, and the possibility that the steroid tapering helped unmasking an underlying RA cannot be completely ruled out. Once RA is 
established, its pathogenic Th1- and Th17-mediated inflammation, in combination with anti-IL-5 therapy, might suppress Th2 cytokine- and eosinophil-mediated inflammation. However, it is also possible that methotrexate, which is sometimes used to treat eosinophilic inflammatory diseases including EGPA, hypereosinophilic syndrome, and eosinophilic fasciitis, ${ }^{12}$ also facilitated discontinuation of benralizumab though the efficacy of MTX for CEP has not been reported before. The optimal treatment duration and criteria for discontinuing biologics in asthma are not yet known and warrant further investigation.

\section{Conclusion}

Anti-IL-5 therapy has various biological effects that could favor a development of arthritis or its flare. The present case may suggest that IL-5 suppression can induce or help unmasking RA in certain patients; however, it is also possible that initial steroid therapy improved subclinical RA and made it remain undiagnosed and the parallel OCS tapering during IL-5 therapy could have contributed to unveil the underlying RA. Regardless, earlier signs of arthritis/morning stiffness should be taken into consideration during anti-IL-5 therapy. Both anti-IL-5 therapy and corticosteroids were discontinued in our patient; however, majority of patients is prescribed prolonged anti-IL-5 therapy because the optimal duration of this therapy is not yet known. Further studies are required to establish guidelines on the optimal use of anti-IL-5 therapy and to understand the possible interactions between CEP, anti-IL -5 therapy, tapering of corticosteroid therapy and development of RA.

\section{Ethics Approval and Informed Consent}

The authors certify that they have obtained written informed consent documents. In the document, the patient agreed with the use of the images and clinical information to be reported in the journal. The patient understood that his name and initials would not be published, and all due efforts would be made to maintain his dignity. This study was approved by the research ethics committee of University of Occupational and Environmental Health, Japan (IRB No. H27-238).

\section{Consent for Publication}

The patient gave written consent to publish this material.

\section{Author Contributions}

All authors significantly contributed to the reported study, whether in the conception, study design, execution, data acquisition, analysis, or interpretation. All authors participated in the drafting, revising, and critical review of the article, gave their final approval for the version to be published, agreed on the journal to which the article was to be submitted, and agreed to be responsible for all aspects of the work.

\section{Funding}

This research was partly supported by a grant from JSPS KAKENHI grant number JP19K17689 (H.K.).

\section{Disclosure}

KY received a research grant from GlaxoSmithKline (GSK) and lecture fees from AstraZeneca and GSK. The other authors have no conflicts of interest for this work.

\section{References}

1. Harish A, Schwartz SA. Targeted anti-IL-5 therapies and future therapeutics for hypereosinophilic syndrome and rare eosinophilic conditions. Clin Rev Allergy Immunol. 2020;59(2):231-247. doi:10.1007/s12016-019-08775-4

2. Chen Z, Bozec A, Ramming A, Schett G. Anti-inflammatory and immune-regulatory cytokines in rheumatoid arthritis. Nat Rev Rheumatol. 2019;15(1):9-17. doi:10.1038/s41584-018-0109-2

3. Suzuki Y, Suda T. Eosinophilic pneumonia: a review of the previous literature, causes, diagnosis, and management. Allergol Int. 2019;68 (4):413-419. doi:10.1016/j.alit.2019.05.006

4. Chen Z, Andreev D, Oeser K, et al. Th2 and eosinophil responses suppress inflammatory arthritis. Nat Commun. 2016;7(1):11596. doi:10.1038/ncomms11596

5. Andreev D, Liu M, Kachler K, et al. Regulatory eosinophils induce the resolution of experimental arthritis and appear in remission state of human rheumatoid arthritis. Ann Rheum Dis. 2020;80(4):451-468. doi:10.1136/annrheumdis-2020-218902

6. Khatri S, Moore W, Gibson PG, et al. Assessment of the long-term safety of mepolizumab and durability of clinical response in patients with severe eosinophilic asthma. J Allergy Clin Immunol. 2019;143 (5):1742-1751. doi:10.1016/j.jaci.2018.09.033

7. Liu W, Ma X, Zhou W. Adverse events of benralizumab in moderate to severe eosinophilic asthma: a meta-analysis. Medicine. 2019;98 (22):e15868. doi:10.1097/MD.0000000000015868

8. Roufosse FE, Kahn JE, Gleich GJ, et al. Long-term safety of mepolizumab for the treatment of hypereosinophilic syndromes. $J$ Allergy Clin Immunol. 2013;131(2):461-467. doi:10.1016/j. jaci.2012.07.055

9. Yamada H, Hida N, Kurashima Y, et al. A case of severe eosinophilic asthma and refractory rheumatoid arthritis well controlled by combination of IL-5Ralpha antibody and TNFalpha inhibitor. Allergol Int. 2019;68(4):536-538. doi:10.1016/j.alit.2019.04.003

10. Tsukamoto N, Takahashi N, Itoh $\mathrm{H}$, et al. Pharmacokinetics and pharmacodynamics of mepolizumab, an anti-Interleukin 5 monoclonal antibody, in healthy Japanese male subjects. Clin Pharmacol Drug Dev. 2018;5(2):102-108. doi:10.1002/cpdd.205 
11. Quirke AM, Perry E, Cartwright A, et al. Bronchiectasis is a model for chronic bacterial infection inducing autoimmunity in rheumatoid arthritis. Arthritis Rheumatol. 2015;67(9):2335-2342. doi:10.1002/ art.39226
12. Groh M, Pagnoux C, Baldini $\mathrm{C}$, et al. Eosinophilic granulomatosis with polyangiitis (Churg-Strauss) (EGPA) Consensus Task Force recommendations for evaluation and management. Eur J Intern Med. 2015;26(7):545-553. doi:10.1016/j.ejim.2015.04.022

\section{Publish your work in this journal}

The Journal of Asthma and Allergy is an international, peer-reviewed open-access journal publishing original research, reports, editorials and commentaries on the following topics: Asthma; Pulmonary physiology; Asthma related clinical health; Clinical immunology and the immunological basis of disease; Pharmacological interventions and

Submit your manuscript here: https://www.dovepress.com/journal-of-asthma-and-allergy-journal new therapies. The manuscript management system is completely online and includes a very quick and fair peer-review system, which is all easy to use. Visit http://www.dovepress.com/testimonials.php to read real quotes from published authors. 